\title{
Validação da determinação de deslocamentos relativos em barragens utilizando Topografia e Medidores Triortogonais de Junta
}

\author{
Validation of the determination of relative displacements on Dams using Topographic Survey \& Joint Gages
}

\author{
Niarkios Luiz Santos de Salles Graça ${ }^{1}$ \\ Pedro Luis Faggion ${ }^{1}$
}

\author{
${ }^{1}$ Departamento de Geomática, Universidade Federal do Paraná - UFPR, Curso de Pós-Graduação em Ciências \\ Geodésicas - CPCG. Avenida Coronel Francisco Heráclito dos Santos, 210 Centro Politécnico - Jardim das Américas \\ Caixa Postal 19001, 81.531-990 Curitiba, Paraná, Brasil. \\ niarkiosnl@hotmail.com, faggion@ufpr.br
}

Recebido em 03/02/2016 - Aceito em 28/06/2016

Received on 03/02/2016 - Accepted on 28/06/2016

\begin{abstract}
RESUMO - O presente estudo apresenta uma metodologia para o levantamento de pontos de monitoramento, internos a galeria de inspeção, instalados junto à instrumentação de auscultação de uma barragem de concreto. A validação da determinação de deslocamentos relativos entre os blocos da barragem, derivados das observações topográficas, é feita utilizando dados oriundos dos medidores triortogonais de junta. Por conveniência, o levantamento topográfico é feito usando um referencial local, vinculado a uma rede geodésica de monitoramento, materializada através de pilares de concreto, engastados na rocha, a montante e a jusante da barragem. Para o conjunto de dados coletados e com base nos processamentos e análises realizadas, os deslocamentos determinados por técnica topográfica apresentaram magnitudes incompatíveis com os valores adotados como referência.
\end{abstract}

Palavras chave: monitoramento, barragem, topografia, medidores triortogonais de junta.

ABSTRACT - This study presents a methodology for the survey of monitoring points, internal to an inspection gallery and installed along the monitoring instrumentation of a concrete dam. Validation of relative displacements between the dam blocks, derived from topographical observations, is done using data from joint gages. For convenience, the survey is done using a local reference system, linked to a monitoring geodesic network, materialized by concrete pillars, embedded the bedrock, upstream and downstream of the dam. For the collected data and based on processing and analysis done, the displacements determined by topographic technique showed magnitudes incompatible with the values adopted as a reference.

Key words: Monitoring, Dam, survey, Joint Gages

\section{INTRODUÇÃO}

No escopo da engenharia, dada à magnitude das obras criadas e riscos inerentes, passou a existir não apenas a necessidade de acompanhamento de todo o processo de criação, mas também da implantação e operação ao longo da vida útil destas obras. Com isso, objetiva-se verificar as reais condições daquilo que se constrói em face do que havia sido planejado, levandose em conta uma avaliação temporal do comportamento destas obras. Assim, o monitoramento de estruturas torna-se necessário na construção civil, principalmente em obras que visam suprir a demanda gerada por recursos energéticos. Cruz (2009), afirma que nos últimos tempos tem havido uma crescente valoração nacional e internacional, dos sistemas de inspeção e observação de obras de engenharia civil, especialmente para barragens. Estas estruturas sofrem com a exposição às intempéries e degradação dos materiais, implicando na alteração das condições de segurança do empreendimento. O mesmo autor afirma ainda que o engenheiro dispõe de inspeções visuais, auscultação geodésica e instrumentação de auscultação como principais meios de avaliar a segurança de um empreendimento.

Suci e Faggion (2012) reforçam esta ideia afirmando que barragens são monitoradas a partir de métodos geodésicos e geotécnicos, ambos se tornam complementares, visto que o primeiro fornece informações de forma global em relação a um sistema de referência pré-definido e o segundo oferece maior precisão, mas sem a vantagem de ter um sistema de referência fora da estrutura e sem a influência regional da massa de água contida no reservatório da barragem.

Casos onde houve rompimento de barragens ou registros de trincas e rupturas tornam-se exemplos de aprendizagem. No cenário nacional cita-se o trágico exemplo da ruptura da barragem de Fundão em Minas Gerais, considerado um dos maiores desastres ambientais da historia do país. Sendo assim, o 
monitoramento desempenha um papel importante no projeto, nos processos construtivos e na operação da Usina.

A instrumentação de uma barragem torna-se ferramenta não apenas para a avaliação das condições de segurança do empreendimento em todas as suas fases, mas também para verificação de hipóteses adotadas em projetos, com o objetivo principal de tornar as obras mais econômicas, dentro das necessárias condições de segurança. A instrumentação deve ser orientada para questões específicas ou para atender critérios de projeto.

Em barragens de concreto, a detecção de eventuais deslocamentos diferenciais entre os blocos que a compõe, pode ser realizada através de leituras em instrumentos específicos. Dentre eles, citam-se os medidores tipo triortogonais e pêndulos (diretos ou invertidos) que permitem a detecção de abertura/fechamento das juntas, recalque diferencial entre blocos e deslizamentos horizontais. Estes instrumentos permitem um controle quase completo dos deslocamentos verticais e horizontais da barragem, em relação a pontos de referência locais (deslocamentos relativos). Silveira (2003) salienta que as técnicas citadas não fornecem deslocamentos absolutos, visto que estes só podem ser obtidos através de medições geodésicas. Porém, o mesmo autor esclarece que a instrumentação dos blocos constitui um método adequado para auscultação de barragens.

Durante a análise dos resultados da instrumentação de uma barragem, as medições dos deslocamentos horizontais e verticais devem ser consideradas simultaneamente, mesmo que obtidas por meios diferentes, por exemplo, instrumentos de monitoramento e topografia (CRUZ, 1996).

Neste sentido, justifica-se investigar uma metodologia para determinação de deslocamentos tridimensionais que possa fornecer informações de caráter local (deslocamentos relativos) ou se desejado, deslocamentos absolutos (geodésicos). Analises de cunho absoluto, não serão abordados neste trabalho.

Teixeira (2005) desenvolveu um trabalho de análise geodésica de deformação de crosta a partir de deslocamentos tridimensionais obtidos pelo sistema de posicionamento global na barragem de Salto Caxias. No mesmo ano, Granemann (2005), desenvolveu pesquisa sobre o desenvolvimento de uma rede geodésica para monitoramento da estrutura da mesma barragem. Já Martini (2005) pesquisou sobre a calibração de prismas de reflexão total em forma de fita adesiva e seu uso no controle de deslocamentos em barragens. Zocolotti (2005) investigou sobre a utilização de poligonais de precisão para o monitoramento bidimensional de pontos dentro da galeria de inspeção de uma barragem. Nadal (2008) pesquisou sobre controle e operação remota de estações totais robotizadas, voltado a auscultação geodésica. Neves (2008) conduziu estudo sobre processamento e análises de levantamentos geodésicos aplicados ao monitoramento de estruturas. Muguio
(2012) conduziu estudo sobre monitoramento continuo por GNSS em uma barragem. Silva (2012) estudou o uso de nivelamento trigonométrico para avaliação de recalques em barragens. Lenartovicz (2013) avaliou a potencialidade do laser scanner terrestre no monitoramento de estruturas. Siguel (2013) estudou o uso da técnica de irradiação tridimensional para monitoramento de pontos afixados em uma barragem.

O presente estudo apresenta uma metodologia para o levantamento de pontos de monitoramento instalados junto à instrumentação de auscultação de uma barragem de concreto e a validação da determinação de deslocamentos relativos tridimensionais (locais) entre os blocos, utilizando dados oriundos dos medidores triortogonais de junta e observações topográficas. Por conveniência e facilitação à interpretação dos resultados, o levantamento topográfico é feito usando um referencial local, vinculado a uma rede geodésica de monitoramento materializada através de pilares de concreto, engastados na rocha, a montante e a jusante da barragem.

\section{REVISÃO DE LITERATURA}

\subsection{Segurança}

Castro et al. (2011), fazendo um breve retrospecto, mencionam que a Comissão Brasileira de Grandes Barragens (CBGB) criada em 1936, seguindo a tendência mundial da década de 70, editou em 1979 e 1983 as "Diretrizes para a Inspeção e Avaliação da Segurança de Barragens em Operação”. Posteriormente em 1986, editou as "Recomendações para a Formulação e Verificação de Critérios e Procedimentos de Segurança de Barragens", em 1995 o "Cadastro Brasileiro de Deterioração de Barragens e Reservatórios” e em 1996, “Auscultação e Instrumentação de Barragens no Brasil". Estas publicações elaboradas por comissões da CBGB serviram para balizar os procedimentos de segurança adotados por algumas organizações brasileiras. Com isso, teve início no país uma discussão sobre a segurança das barragens.

\subsection{Auscultação Geodésica de barragens}

O termo "Auscultação", comum da medicina, vem a tempos sendo empregado na construção civil com vistas ao monitoramento. Neste sentido, o termo pode ser entendido como um conjunto de formas de observação do comportamento da barragem e fundações, para controlar suas condições de segurança, comprovar a validade das hipóteses e dos métodos de cálculos utilizados no projeto e verificar a necessidade de ações corretivas (ITAIPU, 2015).

Medições geodésicas de recalques em barragem, portanto utilizando técnicas absolutas, foram utilizadas pela primeira vez na barragem suíça de Montsalvens em 1921, generalizando-se entre as barragens 
europeias e americanas nas décadas seguintes (SILVEIRA, 2003).

Levantamentos geodésicos e topográficos têm sido tradicionalmente empregados para determinar deslocamentos de pontos na superfície de um objeto em relação a outros tidos como estáveis, conforme cita o Department Of The Army (1994). Na mesma publicação, menciona-se ainda que quando os pontos de referência estão localizados na própria estrutura apenas deformações relativas podem ser determinadas. Chen (1983) cita as seguintes razões para o uso de métodos geodésicos em levantamentos de deformação:

(a) Fornecem o status global para todo o corpo deformável;

(b) Contém o esquema de auto checagem dos resultados e são capazes de avaliar a acurácia das medições globalmente;

(c) Fornecem versatilidade e conveniência para qualquer ambiente e condição de operação;

\subsection{Instrumentação}

Cruz (2009) esclarece que a segurança de uma barragem depende fundamentalmente do projeto de construção e que a instrumentação constitui um método de observação muito importante, mas não aumenta intrinsecamente a segurança da obra. Aponta ainda que os principais objetivos da instrumentação de uma barragem são:

(a) Verificar as hipóteses, os critérios e os parâmetros adotados em projeto, de modo a permitir o aprimoramento do projeto da própria obra em estudo, ou de futuras barragens, visando a condições mais econômicas e/ou mais seguras;

(b) Verificar a adequação de métodos construtivos;

(c) Verificar as condições de segurança das obras, de modo a serem adotadas medidas corretivas em tempo hábil, se necessárias;

Os medidores triortogonais de junta (MTJ), são usualmente empregados para monitorar fissuras, trincas ou juntas de contração entre blocos. Através deles, podem-se determinar deslocamentos diferenciais entre blocos segundo três direções ortogonais entre si (recalques diferenciais, deslocamentos horizontais e movimento de abertura e fechamento da junta) Fig. 1.

A auscultação de estruturas de concreto de uma barragem, através de observações de deslocamentos diferenciais entre blocos, é um procedimento usual para detectar eventuais anomalias de comportamento. Matos (2002) descreve que o princípio de funcionamento de um MTJ consiste em medir deslocamentos em três direções ortogonais, com o auxílio de um sistema de apoios construídos em aço inoxidável e fixados convenientemente na estrutura. Com relógio comparador ou paquímetro, são feitas as medidas nos três eixos ortogonais desejados, e quando se compara as leituras entre as épocas observadas, eventuais deslocamentos são traduzidos em variações nas leituras. Silveira (2003), menciona que o relógio comparador usado para este fim deve apresentar campo de leitura da ordem de $10 \mathrm{~mm}$ (ou mais) e sensibilidade de 1 mícron $(0,001 \mathrm{~mm})$. Afirma ainda que tal instrumento, desde que operado por pessoas cuidadosas e de bom nível técnico, permite uma precisão da ordem de $\pm 0,01 \mathrm{~mm}$.

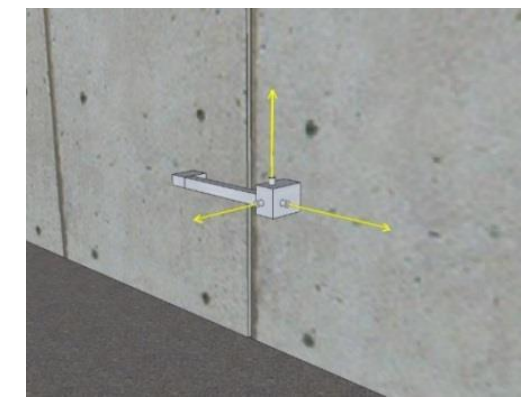

Figura 1 - medidor tipo triortogonal de junta e orientação dos respectivos eixos.

\section{MATERIAIS E MÉTODOS}

A investigação proposta foi conduzida na Barragem da Usina Hidrelétrica Mauá. Esta se localiza no trecho médio do Rio Tibagi, afluente pela margem esquerda do rio Paranapanema, na divisa dos municípios de Telêmaco Borba e Ortigueira, no Estado do Paraná. A referida barragem é de concreto compactado com rolo (CCR) com $745 \mathrm{~m}$ de comprimento na crista e $85 \mathrm{~m}$ de altura máxima.

Foram realizadas cinco campanhas de coleta de dados entre as dadas 17/12/2012 e 21/11/13, contemplando tanto as fases de inicio do enchimento do reservatório da barragem e o inicio da operação da usina, bem como a variação das estações do ano (inverno e verão).

As observações realizadas foram feitas empregando-se uma estação total robotizada Leica modelo TCR 1205 com precisão angular de 5 segundos. Todos os procedimentos de verificação e retificação ou calibração foram rigorosamente realizados imediatamente antes de cada campanha de coleta de dados com auxilio de colimadores em laboratório (DA SILVA et al, 2010). Após estes procedimentos e novas verificações, obteve-se que os valores dos desvios padrão para observações angulares (horizontais e verticais) foram inferiores a 2 segundos de arco.

\subsection{Sistemas de Referência}

Com o intuito de facilitar o entendimento e a interpretação visual dos resultados, por parte do corpo técnico que administra a barragem e dos autores, foi proposto e implementado um sistema de referência cartesiano local onde o eixo " $\mathrm{x} "(\mathrm{Xb})$ estivesse no sentido longitudinal da estrutura da barragem, com orientação positiva na direção da margem direita, o 
eixo "y" (Yb) perpendicular a este e orientação positiva no sentido do fluxo do rio (jusante) enquanto que o eixo " $\mathrm{z}$ " ( $\mathrm{Zb})$ completa o terno do sistema, positivo na direção do zênite. Este sistema foi derivado de um Sistema Geodésico Cartesiano Local - SGCL (ANDRADE, 2003) cuja origem foi arbitrada no pilar PG02 que compõe a Rede Geodésica de Monitoramento existente na área (SIGUEL, 2013 e DA CRUZ, 2015). A Erro! Fonte de referência não encontrada. ilustra a rede geodésica mencionada (PG01, PG02, PG03 e CG 01) e os sistemas de referência adotados (SGCL, em azul e SB em vermelho).

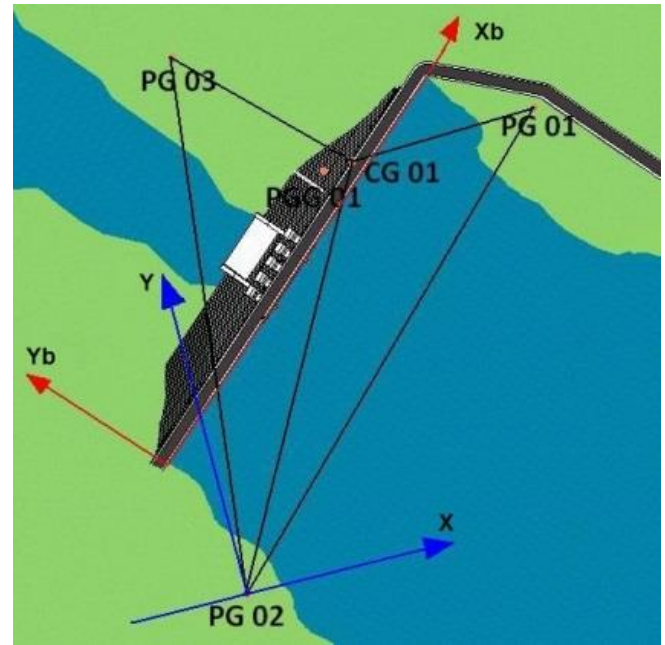

Figura 2 - Rede geodésica de monitoramento e sistemas de referência.

Por questões de simplificação, é apresentada apenas a metodologia de transformação do SGCL com origem no PG02 para o referencial proposto, denominado de Sistema da Barragem-SB (também cartesiano de ordem local). Uma vez calculadas as coordenadas de todos os pilares da rede e de pontos que definem a origem e as orientações dos eixos do novo sistema (SB) no SGCL, aplica-se uma translação e uma rotação ativa (LUGNANI, 1987) no eixo " $z$ " com amplitude $\theta$, conforme o modelo a seguir:

$$
\begin{aligned}
& {\left[\begin{array}{c}
\mathrm{x}_{\mathrm{b}} \\
\mathrm{y}_{\mathrm{b}} \\
\mathrm{z}_{\mathrm{b}}
\end{array}\right]=\mathrm{R}^{-1}\left[\begin{array}{c}
\mathrm{x}-\mathrm{x}_{0} \\
\mathrm{y}-\mathrm{y}_{0} \\
\mathrm{z}-\mathrm{z}_{0}
\end{array}\right]} \\
& \mathrm{R}=\left[\begin{array}{ccc}
\cos \theta & -\operatorname{sen} \theta & 0 \\
\operatorname{sen} \theta & \cos \theta & 0 \\
0 & 0 & 1
\end{array}\right] \\
& \theta=\frac{\mathrm{y}-\mathrm{y}_{0}}{\sqrt{\left(\mathrm{x}-\mathrm{x}_{0}\right)^{2}+\left(\mathrm{y}-\mathrm{y}_{0}\right)^{2}}}
\end{aligned}
$$

Onde:

$\mathrm{x}_{\mathrm{b}}, \mathrm{y}_{\mathrm{b}}, \mathrm{z}_{\mathrm{b}}$ - coordenadas cartesianas no sistema da barragem.

$\theta$ - ângulo de rotação entre os sistemas. $\mathrm{x}_{0}, \mathrm{y}_{0}, \mathrm{z}_{0}$ - coordenadas do ponto origem no SGCL.

$\mathrm{x}, \mathrm{y}, \mathrm{z}$ - coordenadas no SGCL do ponto a ser transformado.

Desta forma, as coordenadas dos pilares que compõe a rede geodésica de monitoramento, passam a ser conhecidas com respeito ao novo referencial estabelecido, permitindo assim que quaisquer pontos levantados a partir destes estejam no novo referencial. Outro aspecto importante deste novo referencial de cunho local é o fato do mesmo estar diretamente vinculado a um referencial geodésico.

\subsection{Poligonação}

Foi estabelecida uma poligonal para densificar os vértices (estações) que, através da técnica de irradiação, servem de apoio para o levantamento de pontos de monitoramento. Estes, definidos por suportes metálicos junto aos MTJ. Esta poligonal contém um dos pilares da rede geodésica local, (PG03) o qual permite visada de orientação em outros pilares da mesma rede (PG01 e CG01). Compreende um perímetro total de aproximadamente $1300 \mathrm{~m}$ sendo um trecho interno à galeria de inspeção. É composta por 17 vértices, sendo 15 no corpo da barragem e a partir dos quais são levantados 45 pontos de monitoramento ao longo de toda a galeria. Na Erro! Fonte de referência não encontrada. pode ser visto dois pontos de monitoramento materializados por prismas refletores (em vermelho) instalados junto a um MTJ entre blocos 10 e 11 . Um prisma refletor em cada bloco.

Para o levantamento da poligonal e dos pontos de monitoramento, foram feitas seis séries de medidas (posição direta e inversa da luneta) para cada direção observada (ré, vante e irradiação).

Após serem determinadas as coordenadas tridimensionais de todos os pontos de monitoramento instalados junto aos MTJ, os pares correspondentes a cada MTJ foram identificados e então calculados os vetores conforme a equação 25 .

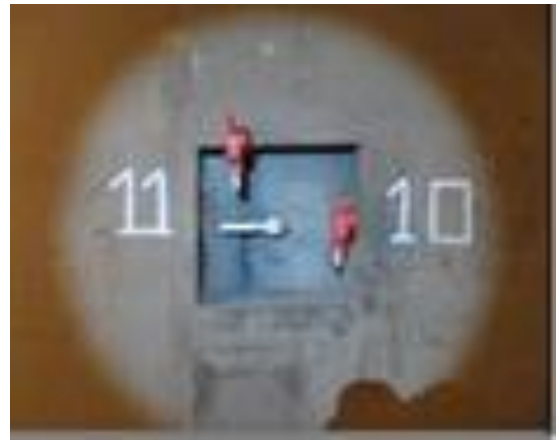

Figura 3 - Pontos de monitoramento junto a um MTJ.

\subsubsection{Cálculos Planimétricos}

A poligonal foi calculada empregando a Regra de Compass (ou Método de Bowditch) (CARVALHO et 
al, 2011), havendo apenas uma distribuição igualitária do erro de fechamento angular. Posteriormente, as coordenadas planimétricas obtidas foram empregadas como valores de aproximação em um ajustamento pelo método de variação de coordenadas. Este ajustamento foi feito com auxilio do Software educacional de ajustamento WOLF PAC@ v.7.1.2 -2013.

No intuito de verificar eventuais erros de observação no ajustamento, Wolf e Ghilani (2007) recomendam que após o ajustamento seja realizada uma análise da presença de eventuais erros grosseiros nas observações. Para tanto, fez- se uma avaliação dos resíduos padronizados fornecidos no arquivo de saída. Foi calculado o valor da estatística $\mathrm{t}$ para cada observação, submetendo-o a um critério de rejeição tabelado com um nível de significância $\alpha=5 \%$ e (r-1) graus de liberdade (sendo " $\mathrm{r}$ " o numero de vértices da poligonal/observações), conforme:

$$
\begin{gathered}
\mathrm{t}=\frac{\overline{\mathrm{v}}}{\hat{\sigma}^{2}} \\
\mathrm{t}>\hat{\sigma}^{2} \mathrm{t}_{\mathrm{tab}}
\end{gathered}
$$

Onde:

$\overline{\mathrm{V}}$ - é o resíduo padronizado, fornecido.

$\hat{\sigma}^{2}$ - variância a posterióri do ajustamento, fornecida.

$\mathrm{t}$ - valor da estatística calculada.

$\mathrm{t}_{\text {tab }}$ - valor de $\mathrm{t}$ tabelado para o nível de significância desejado.

Sendo assim, rejeitam-se todas as observações correspondentes aos valores que forem maiores que $\hat{\sigma}^{2} t_{\text {tab }}$ e um novo ajustamento é realizado. Esta análise é repetida eliminando-se uma observação por vez, reaplicando o ajustamento até que todas as observações sejam aprovadas no teste.

\subsubsection{Cálculos Altimétricos}

Os cálculos altimétricos foram conduzidos de forma independente da planimetria onde a partir dos valores médios dos ângulos zenitais, distâncias inclinadas e respectivos desvios-padrão nas posições de ré e vante, é possível ajustar os desníveis entre os vértices da poligonal.

A determinação dos desníveis é feita de forma indireta através da seguinte equação:

$$
\Delta \mathrm{h}_{\mathrm{AB}}=\text { hi-hs }+ \text { di } \cos (\mathrm{z})
$$

Onde:

$\Delta \mathrm{h}_{\mathrm{AB}}$ - Desnível do ponto "A" para o ponto "B". di - distância inclinada.

$\mathrm{z}$ - ângulo zenital.

hi - altura do instrumento em "A".

hs - altura do sinal "B".

Em uma poligonal fechada, o número de desníveis será igual ao número de vértices. Porém, ao longo do caminhamento existem observações para as direções de ré e de vante. Portanto, para cada estação ocupada haverá condição de determinação do desnível para a direção de ré e de vante.

Conhecendo-se a altitude das estações, o desnível da estação "ré" em relação à estação "vante" é dado pela diferença de altitude:

$$
\Delta \mathrm{h}_{\mathrm{R}_{-} \mathrm{V}}=\mathrm{H}_{\mathrm{V}}-\mathrm{H}_{\mathrm{R}}
$$

Então, assume-se que:

$$
\begin{gathered}
\Delta h_{R_{-} V}=\left(h i-h s_{V}+\left(d i d_{V} \cos \left(z_{V}\right)\right)-\right. \\
\left(h i-h s_{R}+\left(d_{R_{R}} \cos \left(z_{R}\right)\right)\right)
\end{gathered}
$$

Uma vez que a altura do instrumento é a mesma para ambas as direções, podemos excluí-la da equação:

$$
\Delta \mathrm{h}_{\mathrm{R}_{-} \mathrm{V}}=\left(\mathrm{di}_{\mathrm{V}} \cos \left(\mathrm{z}_{\mathrm{V}}\right)-\mathrm{hs}_{\mathrm{V}}\right)-\left(\mathrm{di}_{\mathrm{R}} \cos \left(\mathrm{z}_{\mathrm{R}}\right)-\mathrm{hs}_{\mathrm{R}}\right)
$$

Da equação anterior, tem-se que o desnível passa a ser função das observações de distância inclinada, ângulo zenital e altura do sinal. Percebe-se que se as alturas dos sinais em "ré" e "vante" forem as mesmas, a contribuição desta variável na equação será anulada.

Entretanto, o levantamento de uma poligonal requer observações não apenas das direções de vante (equação 06), mas também de direções de ré. Portanto, ao ocupar um vértice da poligonal são tomadas leituras na direção do vértice a ré e também na direção do vértice de (vante). Neste caso emprega-se a equação 09 que permite determinar o desnível entre os vértices à ré e à vante. Em outras palavras, tendo-se percorrido sequencialmente os vértices da poligonal, pode-se considerar a possibilidade da determinação dos desníveis entre os vértices empregando num primeiro momento somente leitoras da direção de vante e num segundo momento os desníveis compreendidos pelos trechos Ré-Vante, utilizando as leituras de ré e vante para o trecho.

Pode-se então ajustar os desníveis na forma paramétrica $\mathrm{L}_{\mathrm{a}}=\mathrm{F}\left(\mathrm{X}_{\mathrm{a}}\right)$, tendo as equações do modelo matemático funcional escritas da seguinte maneira, para um exemplo de poligonal de três vértices:

$$
\begin{gathered}
\mathrm{F}_{1}: \quad \Delta \mathrm{h}_{1}=\mathrm{l}_{1}+\mathrm{v}_{1} \quad \mathrm{~F}_{3}: \\
\mathrm{F}_{2}: \Delta \mathrm{h}_{2}=\mathrm{l}_{2}+\mathrm{v}_{2} \\
\Delta \mathrm{h}_{3}=\mathrm{l}_{3}+\mathrm{v}_{3} \\
\mathrm{~F}_{4}: \quad \Delta \mathrm{h}_{1}+\Delta \mathrm{h}_{2}=\mathrm{l}_{4}+\mathrm{v}_{4} \\
\mathrm{~F}_{5}: \quad \Delta \mathrm{h}_{2}+\Delta \mathrm{h}_{3}=\mathrm{l}_{5}+\mathrm{v}_{5} \\
\mathrm{~F}_{6}: \quad \Delta \mathrm{h}_{3}+\Delta \mathrm{h}_{1}=\mathrm{l}_{6}+\mathrm{v}_{6}
\end{gathered}
$$

O caso em questão oferece superabundância de observações (trechos "l") em relação ao número de incógnitas ou parâmetros $(\Delta \mathrm{h})$. Isso permite $\mathrm{o}$ ajustamento pelo Método dos Mínimos Quadrados (GEMAEL, 1994) o qual deve atender a seguinte condição:

$$
\sum_{\mathrm{i}=1}^{\mathrm{n}} \mathrm{v}^{2}=\text { mínimo }
$$


A solução do modelo paramétrico de ajustamento é dada pelas equações normais:

$$
\begin{gathered}
X_{a}=\left(A^{T} P A\right)^{-1} A^{T} P L_{b} \\
X_{a}=N^{-1} U
\end{gathered}
$$

Onde:

A - Matriz das derivadas parciais do modelo funcional em relação aos parâmetros.

$\mathrm{L}_{\mathrm{b}}$ - Matriz das observações (desníveis obtidos através das equações 06 e 09).

$\mathrm{P}$ - Matriz dos pesos dada por $\mathrm{P}=1 / \sigma^{2} \mathrm{Lb}$.

Assumindo que as variáveis envolvidas são não correlacionadas, o peso adotado no ajustamento pode ser obtido através da aplicação da Lei de Propagação das Covariâncias, aplicada na forma clássica (equação 14) às equações 06 e 09.

$\sigma_{y}^{2}=\left(\frac{\partial f y}{\partial x_{1}}\right)^{2} \sigma_{x 1}^{2}+\left(\frac{\partial f y}{\partial x_{2}}\right)^{2} \sigma_{x 2}^{2}+\ldots+\left(\frac{\partial f y}{\partial x_{n}}\right)^{2} \sigma_{x n}^{2}$

Onde:

$\sigma^{2}-$ Variância.

fy - Função $y$ qualquer.

$\mathrm{x}_{1}, \mathrm{x}_{2}, \mathrm{x}_{\mathrm{n}}$ - Parâmetros envolvidos.

Tradicionalmente, o erro de fechamento em um circuito de nivelamento é obtido através do somatório dos desníveis. De forma análoga, uma poligonal fechada pode também ser entendida como um circuito fechado de nivelamento, permitindo assim a adoção de uma injunção no ajustamento:

$$
\mathrm{G}\left(\mathrm{X}_{\mathrm{a}}\right)=\Delta \mathrm{h}_{1}+\Delta \mathrm{h}_{2}+\cdots+\Delta \mathrm{h}_{\mathrm{n}}=0
$$

A adoção desta injunção no ajustamento terá como significado prático o efeito de que, após obtidos os desníveis ajustados (parâmetros), a condição imposta pela injunção estará satisfeita, ou seja, a magnitude do erro de fechamento será distribuída e o erro de fechamento será igual a zero.

Para isso, calculam-se novas equações normais para o modelo funcional da injunção e somá-las as equações normais do ajustamento:

$$
\mathrm{X}_{\mathrm{a}}=\left(\mathrm{N}+\mathrm{N}_{\mathrm{c}}\right)^{-1}\left(\mathrm{U}+\mathrm{U}_{\mathrm{c}}\right)
$$

Onde:

$$
\begin{aligned}
& \mathrm{N}_{\mathrm{c}}=\mathrm{C}^{\mathrm{T}} \mathrm{P}_{\mathrm{c}} \mathrm{C} \\
& \mathrm{U}_{\mathrm{c}}=\mathrm{C}^{\mathrm{T}} \mathrm{P}_{\mathrm{c}} \mathrm{E}
\end{aligned}
$$

Sendo: injunção.

E - Vetor erro de fechamento do funcional da

C - Derivadas parciais de $\mathrm{G}$ em relação aos parâmetros ajustados.
$P_{c}$ - Peso da injunção, geralmente um escalar de magnitude maior que as grandezas mensuradas.

A determinação das altitudes dos vértices da poligonal se dá somando sucessivamente os desníveis ajustados a uma altitude inicial. As precisões das altitudes são obtidas através da aplicação da Lei de Propagação das Covariâncias conforme:

$$
\begin{gathered}
\mathrm{H}_{\mathrm{B}}=\mathrm{H}_{\mathrm{A}}+\Delta \mathrm{h}_{\mathrm{AB}} \\
\sigma_{\mathrm{H}_{\mathrm{B}}}^{2}=\left(\frac{\partial \mathrm{H}_{\mathrm{B}}}{\partial \mathrm{H}_{\mathrm{A}}}\right)^{2} \sigma_{\mathrm{H}_{\mathrm{A}}}^{2}+\left(\frac{\partial \mathrm{H}_{\mathrm{B}}}{\partial \Delta \mathrm{h}_{\mathrm{AB}}}\right)^{2} \sigma^{2}{ }_{\Delta \mathrm{h}_{\mathrm{AB}}}
\end{gathered}
$$

Uma vez determinadas as coordenadas tridimensionais dos vértices da poligonal e suas precisões é possível determinar as coordenadas das irradiações através das equações 21,22 e 23 , e precisões conforme equação 14 .

$$
\begin{gathered}
\mathrm{X}_{\mathrm{IR}}=\mathrm{X}_{\mathrm{A}}+\left(\mathrm{di}_{\mathrm{AIR}} \operatorname{sen}(\mathrm{Z})\right) \operatorname{sen}\left(\mathrm{AZ}_{\mathrm{AIR}}\right) \\
\mathrm{Y}_{\mathrm{IR}}=\mathrm{Y}_{\mathrm{A}}+\left(\mathrm{di}_{\mathrm{AIR}} \operatorname{sen}(\mathrm{Z})\right) \cos \left(\mathrm{AZ} \mathrm{Z}_{\mathrm{AIR}}\right) \\
\mathrm{Z}_{\mathrm{IR}}=\mathrm{Z}_{\mathrm{A}}+\mathrm{hi}-\mathrm{hs}+\left(\mathrm{di}_{\mathrm{AIR}} \cos (\mathrm{Z})\right)
\end{gathered}
$$

As equações acima $(21,22$ e 23$)$ possuem como variáveis de entrada as coordenadas do ponto estacionado $\left(\mathrm{X}_{\mathrm{A}}, \mathrm{Y}_{\mathrm{A}}, \mathrm{Z}_{\mathrm{A}}\right)$, observações de distância inclinada e ângulo vertical para as direções de irradiação $\left(\mathrm{di}_{\mathrm{AIR}}, Z\right)$ e o azimute da direção de irradiação $\left(\mathrm{AZ}_{\mathrm{AIR}}\right)$. Os azimutes das direções das irradiações são obtidos a partir do azimute da direção do lado da poligonal e dos ângulos entre as direções de ré e das direções das irradiações. Os azimutes das direções da poligonal e respectivas precisões, por sua vez, são calculados a partir das coordenadas ajustadas e precisões.

\subsection{Análises dos deslocamentos}

Da teoria da geometria analítica (VENTURI, 1949), tem-se que é possível tratar a diferença de coordenadas entre dois pontos (A e B) no espaço como um vetor:

$$
\begin{gathered}
\overrightarrow{A B}=B-A \\
\overrightarrow{A B}=\underbrace{\left(x_{B}-x_{A}\right)}_{\Delta x} \vec{i}+\underbrace{\left(y_{B}-y_{A}\right)}_{\Delta y} \vec{j}+\underbrace{\left(z_{B}-z_{A}\right)}_{\Delta z} \vec{k}
\end{gathered}
$$

Onde:

$\overrightarrow{\mathrm{AB}}$ - representa o vetor com origem no ponto $\mathrm{A}$ na direção de B.

$\left(\mathrm{x}_{\mathrm{A}}, \mathrm{y}_{\mathrm{A}}, \mathrm{z}_{\mathrm{A}}\right)$ - Coordenadas do ponto $\mathrm{A}$.

$\left(\mathrm{x}_{\mathrm{B}}, \mathrm{y}_{\mathrm{B}}, \mathrm{z}_{\mathrm{B}}\right)$ - Coordenadas do ponto $\mathrm{B}$.

$\left(\Delta_{x}, \Delta_{y}, \Delta_{z}\right)$ - Diferença de coordenadas entre os pontos $\mathrm{A}$ e B.

$\overrightarrow{\mathrm{i}}, \overrightarrow{\mathrm{j}}, \overrightarrow{\mathrm{k}}$ - versores dos eixos cartesianos ortogonais $\mathrm{x}, \mathrm{y}, \mathrm{z}$. 
Nesta abordagem relativa, a cada campanha de coleta de dados calculam-se os vetores distância (espacial) entre os pontos e em campanhas consecutivas avaliam-se eventuais variações de magnitude nas componentes $\overrightarrow{\mathrm{i}}, \overrightarrow{\mathrm{j}}$ e $\overrightarrow{\mathrm{k}}$. Esta abordagem permite uma análise comparativa entre épocas, da variação das componentes do vetor e a respectiva orientação em relação aos eixos. Adotando uma época de referência " $t$ " os deslocamentos relativos obtidos pela abordagem topográfica $\left(D_{R n}\right)$ e via MTJ $\left(D_{\text {Rn(MTJ) }}\right)$, existentes em um local “n”, em relação a uma época "i”, ficam então caracterizados por eventuais variações das componentes vetoriais $\left(\Delta_{\mathrm{x}} \overrightarrow{\mathrm{i}}, \Delta_{\mathrm{y}} \overrightarrow{\mathrm{j}}, \Delta_{\mathrm{z}} \overrightarrow{\mathrm{k}}\right)$ e pelas variações das leituras tomadas nos $\operatorname{MTJ}\left(\mathrm{L}_{\mathrm{x}}, \mathrm{L}_{\mathrm{y}}, \mathrm{L}_{\mathrm{z}}\right)$ conforme:

$$
\begin{aligned}
& D_{\text {Rn }}=\left(\Delta_{x} \vec{i}, \Delta_{y} \vec{j}, \Delta_{z} \vec{k}\right)_{t+i}-\left(\Delta_{x} \vec{i}, \Delta_{y} \vec{j}, \Delta_{z} \vec{k}\right)_{t} \\
& D_{\text {Rn(MTJ) }}=\left(L_{x}, L_{y}, L_{z}\right)_{t+i}-\left(L_{x}, L_{y}, L_{z}\right)_{t}
\end{aligned}
$$

\subsection{Validações dos deslocamentos}

A validação dos deslocamentos obtidos pela abordagem topográfica $\left(\mathrm{D}_{\mathrm{Rn}}\right)$ frente aos deslocamentos derivados das leituras nos $\mathrm{MTJ}\left(\mathrm{D}_{\mathrm{Rn}(\mathrm{MTJ})}\right)$ pressupõe a equivalência dos valores obtidos por ambas. O teste de Fisher é tradicionalmente empregado para avaliar a igualdade das variâncias entre amostras a um nível de significância $\alpha$. No entanto, este teste assume a existência de graus de liberdade no numerador e no denominador para o cálculo de um valor teórico, o qual será confrontado com um valor tabelado para os graus de liberdade em questão. Esta condição de existência de graus de liberdade não é satisfeita no presente trabalho, pois para cada época a ser confrontada (validada) e para o mesmo ponto sendo avaliado, há apenas um único valor de deslocamento para cada método (Topografia e MTJ), implicando portanto em graus de liberdade iguais a zero.

Entretanto, uma análise visual dos dados pode ser feita através do uso do conceito conhecido como "BoxPlot" ou diagrama de caixa, comum em análise exploratória de dados e estatística descritiva. Neste tipo de gráfico, os dados são descritos através dos elementos: mediana, primeiro quartil (ou quartil inferior - Q1), terceiro quartil (ou quartil superior Q3), valor máximo e valor mínimo. De acordo com Araujo e Abar (2012) o primeiro quartil, a mediana e o terceiro quartil são três valores que dividem os dados ordenados em quatro grupos com aproximadamente $25 \%$ dos valores em cada grupo. Afirmam ainda que na estatística descritiva ou na análise exploratória e comparação de dados, o BoxPlot é um gráfico configurado para poder identificar os outliers (valores discrepantes), no sentido de estarem muito afastados da maioria dos dados. Este gráfico pode ser modificado de forma que os valores mínimos e máximos sejam substituídos pelos valores abaixo do primeiro quartil por uma quantidade que pode ser maior do que $o$ mínimo [Q1-1,5(Q3-Q1)] e uma quantidade que pode ser menor que máximo [Q3+1,5(Q3-Q1)], respectivamente. Por exemplo, a identificação dos valores outliers é importante no cálculo da média aritmética que tem como característica a influência dos valores extremos.

Massart et al. (2005), reafirmam que para a comparação de duas ou mais séries de resultados, são empregados métodos estatísticos clássicos tais como o teste $\mathrm{t}$ para comparação de médias, F-teste para comparação de variâncias de duas séries de dados e análise de variância (ANOVA) mas que estes métodos são vulneráveis à presença de outliers. A representação de vários diagramas (várias amostras) justapostos em uma mesma figura é uma excelente maneira de investigar se há diferenças entre os conjuntos de dados pode ser aplicado sem que nenhuma suposição estatística seja presumida, por exemplo, uma distribuição normal dos dados.

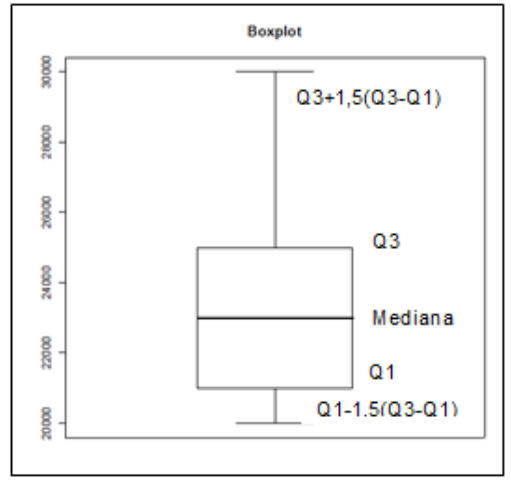

Figura 4 - Exemplo de um Box Plot.

\section{RESULTADOS}

Primeiramente foram determinadas as coordenadas dos pilares da rede geodésica de referência no sistema local proposto (Sistema da Barragem) e assumindo a referida rede como estável, adotaram-se as coordenadas obtidas para a primeira época como referência para os cálculos nas épocas seguintes.

As leituras nos MTJ foram feitas pelo corpo técnico responsável pela operação e manutenção da barragem, fazendo parte da atividade cotidiana de inspeção. Posteriormente foram disponibilizadas para este estudo.

Após minuciosa análise, foi constatado que a época um (primeira campanha) apresentou erro sistemático que impediam seu uso como época de referência a ser empregada na equação 26, sendo então adotada a época dois (segunda campanha). Percebeu-se que a magnitude dos deslocamentos tanto $2 \mathrm{D}$ quanto 3D obtidos por topografia divergiram sobre maneira dos resultados obtidos pelos MTJ. Esta divergência de magnitude de valores sugeriu uma verificação individual da contribuição de cada componente nos deslocamentos espaciais. Percebeu-se que havia indícios do uso inadequado de uma pequena peça de 
adaptação do prisma. A ocorrência desse fato sugeriu a adoção da segunda campanha (05/09/12) como época de referência, sendo realizada aproximadamente 18 dias após a primeira.

Por questão de simplificação, os valores obtidos para as equações 25,26 e 27 serão omitidos e apenas os diagramas de caixa são apresentados.

Considerando a época 2 como referência ("t") e as demais épocas (“i”), aplicou-se as equações 26 e 27 para obtenção dos deslocamentos relativos $2 \mathrm{D}$ e $3 \mathrm{D}$ referentes as épocas $2 \& 3,2 \& 4$ e $2 \& 5$. Para construção dos diagramas de caixa, foram agrupadas as resultantes de todos os deslocamentos relativos, dos três períodos supracitados, em dois conjuntos: MTJ e Topografia. Nas Figs. 5 e 6 são consideradas uma abordagem de análise $2 \mathrm{D}$ (resultante das componentes $\vec{\imath} \mathrm{e} \overrightarrow{\mathrm{j}}$ ) enquanto que nas Figs. 7 e 8 considera-se uma análise 3D (resultante das componentes $\overrightarrow{\mathrm{i}}, \overrightarrow{\mathrm{j}} \mathrm{e} \overrightarrow{\mathrm{k}}$ ). Através da análise do diagrama de caixa, feito para comparar e validar os deslocamentos 2D nota-se o quão disperso os deslocamentos obtidos por topografia estão em relação aos obtidos através de leitura direta nos MTJ. Os pontos mostrados fora dos limites são considerados outliers, destoando dos demais.

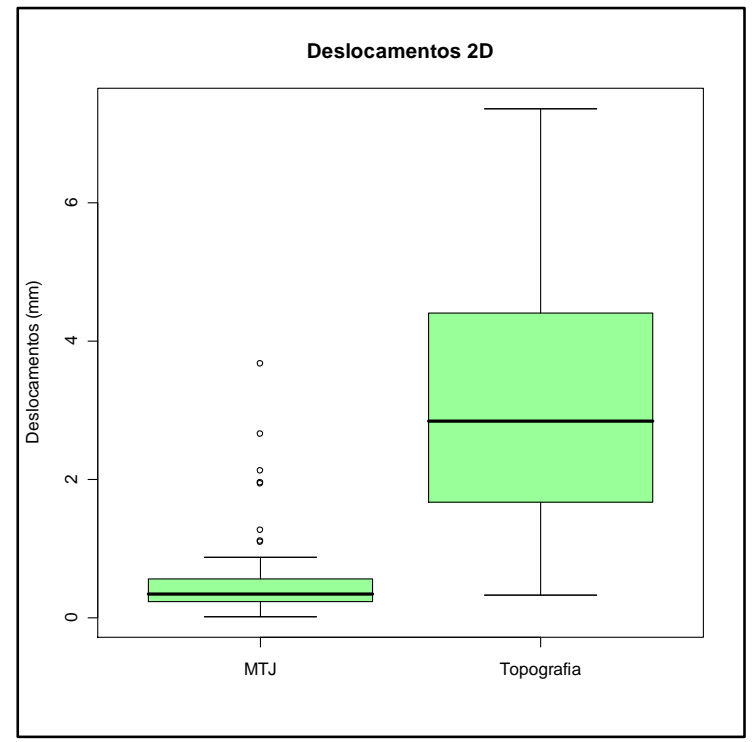

Figura 5 - Box plot para os deslocamentos 2D.

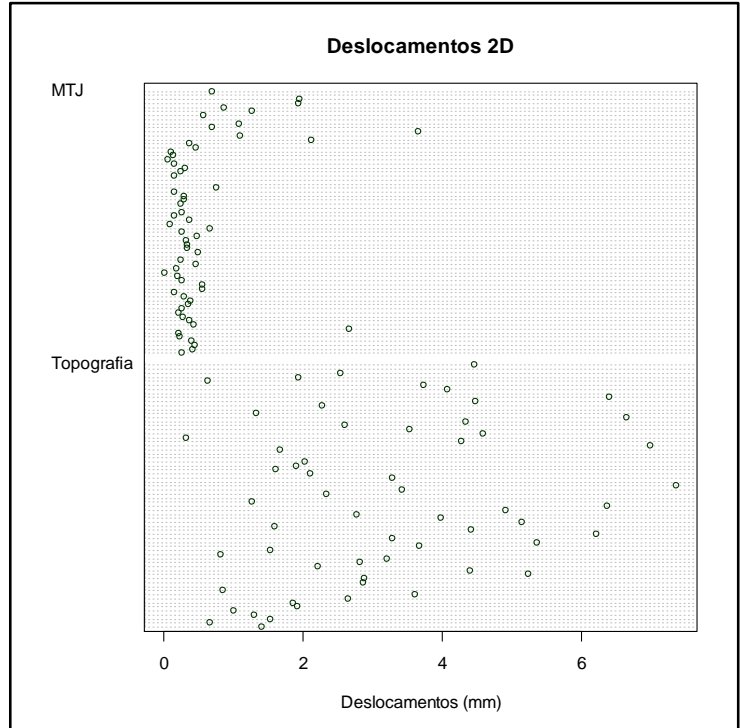

Figura 6 - Diagrama de dispersão pontual dos deslocamentos 2D.

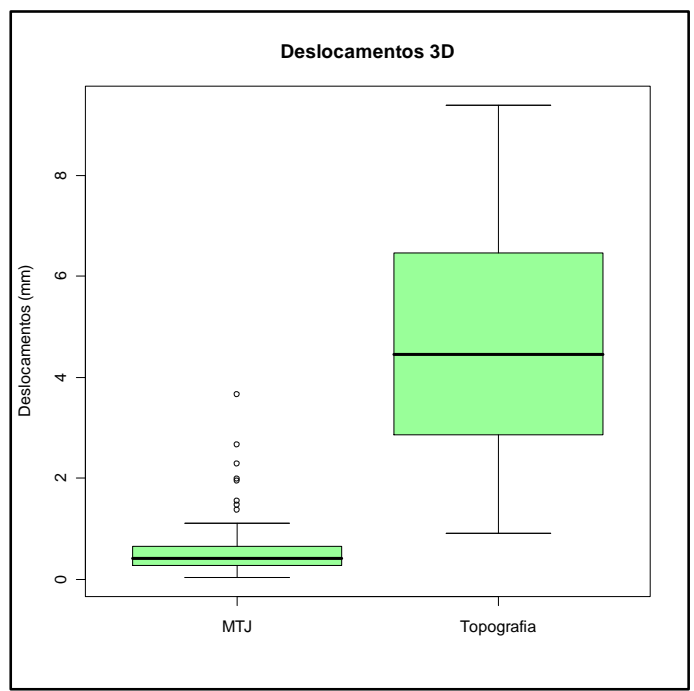

Figura 7 - Box plot para os deslocamentos 3D

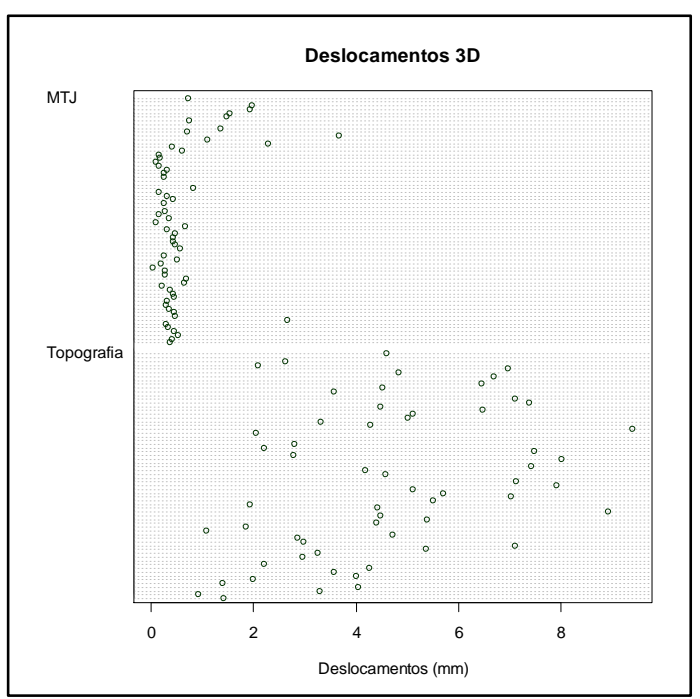

Figura 8- Diagrama de dispersão pontual dos deslocamentos 3D. 
Através dos diagramas construídos, fica evidente que, para os conjuntos de dados analisados, não houve compatibilidade entre os deslocamentos obtidos através das duas técnicas. Nas Figs. 5 e 7, para a amostra Topografia, os afastamentos dos quartis superiores (Q3) e inferiores (Q1) são maiores em relação à amostra MTJ e as medianas apresentam-se com magnitudes em torno de cinco e nove vezes maiores respectivamente, que as magnitudes atingidas para na amostra MTJ (adotados como referência, por serem obviamente mais precisos) e com um afastamento entre Q3 e Q1 menores. Chama-se a atenção para o fato de que os valores classificados como outliers são exclusivamente para as amostras MTJ. São eles: MTJ-8 (2\&3) e MTJ-11, 12, 13, 17, 21, 22, 23, 24 (2\&5). Esta classificação necessariamente pode não estar correta, já que há possibilidades de ter havido de fato uma variação significativa nos valores registrados para as leituras (deslocamento efetivo dos pontos).

Mesmo assim a possibilidade de erros de leitura nos MTJ ou anotação (erro grosseiro) não deve ser sumariamente descartada.

Através das Figs. 6 e 8, nota-se a dispersão pontual dos deslocamentos e uma concentração dos valores das amostras MTJ em relação as amostras Topografia, em torno de um valor central (menor), juntamente com o afastamento dos pontos classificados potencialmente como outliers em relação a este valor central. Fato que não se apresenta nos valores da amostra Topografia já que não há tendência de concentração de valores em torno de um valor central perceptível (comprovado também pelo afastamento entre Q3 e Q1 já citado).

\section{CONCLUSOES}

O presente estudo apresenta uma metodologia para o levantamento topográfico $3 \mathrm{D}$ de pontos de monitoramento instalados junto à instrumentação de auscultação de uma barragem de concreto e a validação da determinação de deslocamentos relativos entre os blocos, utilizando dados oriundos dos medidores triortogonais de junta.

Com base nos dados existentes foi possível concluir que a metodologia não apresentou acuidade suficiente para mensurar deslocamentos com magnitudes detectáveis pelo instrumental de auscultação.

Entretanto, salienta-se que a barragem da UHE Mauá é uma obra de engenharia que estava em faze inicial de operação durante esta pesquisa e alguns ajustes ainda estavam sendo feitos por parte do pessoal de operação da usina. Foram instalados diversos equipamentos dentro da galeria de inspeção, exatamente onde estavam concentrados os pontos de monitoramento investigados neste trabalho. Foi constatado que em alguns locais, os suportes dos prismas que haviam sido instalados para subsidiar esta pesquisa estavam sendo utilizado pelos trabalhadores da barragem para pendurar cabos, ferramentas, mochilas, etc. Alguns desses suportes foram inclusive danificados de forma permanente de forma a inviabilizar o uso neste estudo. As medidas feitas nestes locais foram desconsideradas pelas razões citadas.

Recomenda-se que sejam realizados mais estudos em condições melhores de forma a comprovar as reais limitações da metodologia proposta.

\section{REFERÊNCIAS BIBLIOGRÁFICAS}

ANDRADE,J.B. Fotogrametria - Jose Bittencourt de Andrade. Curitiba; SBEE,2003;2.ed.

ARAUJO, P.C., ABAR, C.A.A.P. Sobre o Boxplot no GeoGebra, $1^{\text {a }}$. Conferência Latino Americana de GeoGebra. ISSN 2237- 9657, pp.13-21, 2012

CARVALHO, Alessandro Salles et al. Utilização da Regra de Compass ou Método de Bowditch e do MMQ no Ajuste de Poligonal Topográfica. Revista Agrogeoambiental, v. 3, n. 1, 2011.

CASTRO,M.M.,MARTINS,J.R.S.,LUCCI,R.M.JACO BSEM,F.R.S. Concepção de um sistema de gerenciamento de ações emergenciais em barragens .XIX Simpósio Brasileiro de Recursos Hídricos.Maceió-AL.2011

CHEN, Y. Analysis of deformation surveys. A generalized method Department of Surveying Engineering Technical, Thesis (Ph.D). University of New Brunswick, Fredericton. New Brunswick, Canada, 1983.

CRUZ, P. T. 100 barragens brasileiras: casos históricos, materiais de construção, projeto. $2^{\mathrm{a}}$ Edição. São Paulo: Oficina de Textos, 1996.

CRUZ, P. T., MATERÓN, B.,FREITAS,M. Barragens de Enroncamento com Face de Concreto. São Paulo: Oficina de Textos, 2009.

DA CRUZ, W. Integração de dados de monitoramento de estruturas antrópicas: estude de caso: UHE Mauá / Wander da Cruz. - Curitiba, 2015. 145 f. : il.

DA SILVA, M. M. S.; FAGGION, P. L.; VEIGA, L. A. K., METODOLOGIA DE CLASSIFICAÇÃO DAS COMPONENTES ANGULARES DE TEODOLITOS E ESTAÇÕES TOTAIS EM LABORATÓRIO. Boletim de Ciências Geodésicas, v. 16, n. 3, 2010

DEPARTMENT OF THE ARMY - U. S. ARMY CORPS OF ENGENEERS. Manual 1110-1-1004 Deformation Monitoring and Control Surveying. Washington, DC, 1994.

GEMAEL,C. Introdução ao ajustamento de observaçãoes: aplicações geodésicas. Camil Gemael. Curitiba:Ed. UFPR, 1994

GRANEMANN, D. C. Estabelecimento de uma rede geodésica para o monitoramento de estruturas: estudo de caso na Usina Hidrelétrica Salto Caxias, Universidade Federal do Paraná, Curitiba, PR, 2005. 
ITAIPU: ENERGIA: 2015. Disponível em: < http://www.itaipu.gov.br/energia/auscultacao > Acessado em 23 de Novembro de 2015

LENARTOVICZ,I. Avaliação da Potencialidade do Laser Scanner Terrestre no monitoramento de estruturas: Estudo de caso Uhe Mauá, Universidade Federal do Paraná, Curitiba, PR, 2013

LUGNANI,J.B. Introdução à Fototriangulação. João Bosco Lugnani. Curitiba,1987. 134 p.

MARTINI,L. Calibração de prismas de reflexão total em forma de fita adesiva, em acrílico e sua aplicação no controle de deslocamentos de estruturas Universidade Federal do Paraná, Curitiba, PR, 2005.

MASSART,D.L., SMEYERS-VERBEKE, J., CAPRON, X., SCHLESIER, K. Visual Presentation of Data by Means of Box Plots. 2005 Disponível em: http://citeseerx.ist.psu.edu/viewdoc/download?doi= 10.1.1.169.9952\&rep=rep1\&type $=$ pdf $>$ Acessado em 20 de Novembro de 2015

MATOS,S.F. Avaliação de instrumentos para auscultação de barragem de concreto. Estudo de caso: deformímetros e tensômetros para concreto na barragem de ITAIPU Dissertação de mestrado Universidade Federal do Paraná, Curitiba, PR, 2002.

MUGUIO,M.R. Implantação e análise da estação GNSS para o monitoramento contínuo da barragem da Usina Hidrelétrica de Mauá . Universidade Federal do Paraná, Curitiba, PR, 2012

NADAL,M.A.D. Controle E Operação Remota De Estações Totais Robotizadas Voltado À Auscultação Geodésica Universidade Federal do Paraná, Curitiba, PR, 2008

NEVES, R. V. Processamento e análise de levantamentos geodésicos aplicado ao monitoramento de estruturas civis. $121 \mathrm{f}$. Universidade Federal do Paraná, Curitiba, PR, 2008

SIGUEL,A.R., Monitoramento da barragem da usina hidrelétrica Mauá utilizando irradiação tridimensional. Dissertação (Mestrado). Curso de Pós Graduação em Ciências Geodésicas, Departamento de Geomática, Universidade Federal do Paraná, Curitiba, PR, 2013

SILVA,R.N.F. Avaliação do método de nivelamento trigonométrico, técnica Leap-Frog, na determinação de recalque em grandes estruturas: estudo de caso para a UHE Mauá. Universidade Federal do Paraná, Curitiba, PR, 2012

SILVEIRA,J. F. A. Instrumentação e comportamento de barragens de concreto / João Francisco Alves Silveira. - São Paulo: Oficina de textos. 2003

SUCI, F. M., \& FAGGION, P. L. (2012). Análise da estabilidade da rede geodésica de monitoramento da UHE Salto Caxias a partir de dados gps. Bol. Ciênc. Geod., sec. Artigos, 18(4), 564-582.

TEIXEIRA, N. N. Análise geodésica de deformações da crosta em regiões de grandes barragens a partir de deslocamentos tridimensionais obtidos pelo sistema de posicionamento global / Niel Nascimento Teixeira. - Curitiba, 2005. xxv, 268 f.: il.; tab.

VENTURI,J.J.,Algebra Vetorial E Geometria Analítica - 8 ed.-CURITIBA, 242 p-1949

WOLF,P.R.,GHILANI,C.D. Adjustment Computations: Statistics and Least Squares in Surveying and GIS. John Wiley \& Sons. 3rd Edition, 1997

ZOCOLOTTI, C.A. Utilização de técnicas de poligonação de precisão para o monitoramento de pontos localizados em galerias de inspeção: estudo de caso da UHE de Salto Caxias. Universidade Federal do Paraná, Curitiba, PR, 2005 